\title{
EDITORIAL Mild narrowing of the cervical carotid is noted
}

\author{
Eric C. Peterson, MD, MS, and Roberto Heros, MD \\ Department of Neurological Surgery, University of Miami Miller School of Medicine, Miami, Florida
}

I $\mathrm{N}$ this issue of the Journal, Scott and the group at University of Texas Southwestern Medical Center study the issue of blunt cervical carotid artery injury. ${ }^{8}$ The introduction of screening protocols with $\mathrm{CT}$ angiography (CTA) has resulted in an increase in the diagnosis of these lesions in trauma patients, and there is a real question of what to do when the radiologist's report reads "mild narrowing of the cervical ICA." The fact that despite a significant amount of literature on the subject the authors chose to study this topic reveals how much uncertainty remains regarding the management of these lesions. They have developed a database of all blunt cervical artery (i.e., carotid and vertebral artery) injuries at their institution. They have named this the Parkland Carotid and Vertebral Artery Injury Survey and are now reporting their results in a series of publications, one for each subgroup of injury in the carotid and vertebral arteries. We would like to make a few points about this paper.

First, it is a large series that is focused on a specific subtype of blunt cerebrovascular injury (BCVI). This is a reasonable approach because $\mathrm{BCVI}$ is a heterogeneous entity. There are significant differences in its natural history depending on the degree and location of the lesion. For starters, the term BCVI technically includes blunt injury to the intracranial vessels, which carries a substantially worse prognosis because the vessels are unsupported in the subarachnoid space and traumatic dissections often lead to catastrophic intracranial hemorrhages and rehemorrhages if not treated adequately. Injury to the extracranial arteries, however, is quite different because the risk of hemorrhage is very low. The principal risk is thromboembolism, and it is in this area that the natural history is uncertain. The risk almost certainly varies with type and severity of injury as well as the artery injured. A commonly cited grading scale attempts to separate these lesions into mild and moderate intimal injuries (less than $25 \%$ and between $25 \%$ and $50 \%$ narrowing, respectively), pseudoaneurysms, and complete occlusions. ${ }^{1}$ Since vertebral and carotid artery injuries seem to behave differently, we are left with essentially 8 different types of cervical artery injuries. Lumping these together in analyses is inappropriate, limiting us to subgroup analyses. Recently, efforts to evaluate these individual injury patterns have begun to be published. Morton et al. ${ }^{7}$ recently published in the Journal their experience focusing only on Grade 4 lesions-complete occlusions. They found a very high stroke rate in carotid artery occlusions (64\%), but a much lower one in vertebral artery occlusions (9\%).

Second, even for the lower-grade injuries, the stroke risk is not insignificant. The authors found a very low stroke rate of $1 \%$ in their series, but this is not in line with the published literature. Most large series published so far have shown substantially higher rates of ischemic events for Grade 1-2 injuries; in the 6\%-10\% range. ${ }^{2,9}$ Of the patients in this series, only $19 \%$ underwent MRI for suspicion of a stroke, and the authors found 1 patient with a stroke thought to be attributable to the lesion. This is probably an underestimation because most of these patients did not have follow-up MR images, and we know that CT scans (which undoubtedly most of these patients had) are not sensitive enough for reliable detection of cerebral infarcts.

Third, it has recently been shown that the stroke risk is highest at the moment of injury, and that many strokes occur before treatment is possible. The Maryland Shock Trauma group recently published their series of patients with BCVI and found that $50 \%$ of the infarctions were detected on admission imaging or occurred within 8 hours, before any antithrombotic therapy could be initiated. ${ }^{9}$ Similarly, the Colorado group found that $90 \%$ of strokes from BCVI occurred before BCVI was diagnosed, ${ }^{2}$ and the Memphis group found that $79 \%$ occurred prior to diagnosis. ${ }^{4}$ Given the systemic bleeding risk from associated injuries in many trauma patients when they first arrive for 
medical attention, blood thinners are often not immediately administered in the hyperacute period. Indeed, of the 18 patients with infarctions in the Maryland series, 15 presented with stroke or had specific contraindications to blood thinners, leaving only 3 patients who could have received anticoagulants before they suffered the cerebral infarct. This begs the question of how effective treatment for these lesions is. Prior studies documenting lower rates of infarction after treatment suffered from substantial selection bias - they classified patients who presented with infarctions under the "no treatment group." In the current study only a patient with bilateral Grade 2 blunt carotid artery injuries suffered a clinically relevant stroke and it occurred shortly after admission, so it is not clear whether anticoagulation would have prevented its occurrence.

Fourth, treatment essentially consists of 3 modalities: antiplatelet therapy with aspirin, anticoagulation with heparin, and endovascular therapy. The latter is rarely indicated for Grade 1-2 lesions, so we will focus on the first 2 options. Traditionally, arterial dissections, spontaneous and traumatic, were believed to be best treated with anticoagulation with heparin. However, recent studies suggest that not only is heparin associated with significant hemorrhagic morbidity in the trauma population, ${ }^{5,6}$ but it also is probably equivalent to antiplatelet therapy with regard to stroke prevention. ${ }^{3,10}$ Given its safer profile and apparently similar efficacy, aspirin has emerged as the preferred treatment choice for low-grade BCVI, although as implied above its value has not been proven and is at best limited, given that such a high proportion of the cerebral infarcts related to these injuries occur before admission.

Finally, these lesions are clearly dynamic and prone to radiographic worsening. The authors of the present study grouped Grades 1 and 2 together and found a 14\% incidence of worsening to pseudoaneurysm or occlusion. Biffl and colleagues performed repeat catheter angiography at 7 days and found that $8 \%$ of Grade 1 lesions progressed to pseudoaneurysms at 7 days, and $45 \%$ of Grade 2 lesions progressed to pseudoaneurysms. ${ }^{2}$ This is a significant proportion of low-grade injuries that continue to dissect into the adventitia, and they clearly warrant early vascular follow-up whether they are treated or not. What to do once a lesion has deteriorated to a pseudoaneurysm is open to debate and beyond the scope of this editorial. Although it is tempting to proceed with endovascular therapy in this situation, it is not clear that this improves outcome. The only 3 patients treated with endovascular stents in this series were treated because of progression to pseudoaneurysm.

In brief, for the all too common patient with multisystem trauma who undergoes a screening CTA scan that documents "mild narrowing of the cervical ICA," starting aspirin when safe and obtaining follow-up vascular imaging at 1 week to confirm radiographic stability seems prudent. Heparin and endovascular stenting should be reserved for lesions that clearly worsen despite antiplatelet therapy. The Southwestern group should be congratulated for keeping this careful prospective registry. We look forward to very important data coming out of this registry in the future.

http://thejns.org/doi/abs/10.3171/2014.7.JNS141247

\section{References}

1. Biffl WL, Moore EE, Offner PJ, Brega KE, Franciose RJ, Burch JM: Blunt carotid arterial injuries: implications of a new grading scale. J Trauma 47:845-853, 1999

2. Biffl WL, Ray CE Jr, Moore EE, Franciose RJ, Aly S, Heyrosa MG, et al: Treatment-related outcomes from blunt cerebrovascular injuries: importance of routine follow-up arteriography. Ann Surg 235:697-707, 2002

3. Cothren CC, Biffl WL, Moore EE, Kashuk JL, Johnson JL: Treatment for blunt cerebrovascular injuries: equivalence of anticoagulation and antiplatelet agents. Arch Surg 144:685690, 2009

4. DiCocco JM, Fabian TC, Emmett KP, Magnotti LJ, Zarzaur BL, Bate BG, et al: Optimal outcomes for patients with blunt cerebrovascular injury (BCVI): tailoring treatment to the lesion. J Am Coll Surg 212:549-559, 2011

5. Eachempati SR, Vaslef SN, Sebastian MW, Reed RL II: Blunt vascular injuries of the head and neck: is heparinization necessary? J Trauma 45:997-1004, 1998

6. Miller PR, Fabian TC, Bee TK, Timmons S, Chamsuddin A, Finkle R, et al: Blunt cerebrovascular injuries: diagnosis and treatment. J Trauma 51:279-286, 2001

7. Morton RP, Hanak BW, Levitt MR, Fink KR, Peterson EC, Vilela MD, et al: Blunt traumatic occlusion of the internal carotid and vertebral arteries. J Neurosurg 120:1446-1450, 2014

8. Scott WW, Sharp S, Figueroa SA, Eastman AL, Hatchette CV, Madden CJ, et al: Clinical and radiographic outcomes following traumatic Grade 1 and 2 carotid artery injuries: a 10-year retrospective analysis from a Level I trauma center. The Parkland Carotid and Vertebral Artery Injury Survey. J Neurosurg [epub ahead of print March 20, 2015. DOI: 10.3171/2015.1.JNS14642]

9. Stein DM, Boswell S, Sliker CW, Lui FY, Scalea TM: Blunt cerebrovascular injuries: does treatment always matter? J Trauma 66:132-144, 2009

10. Wahl WL, Brandt MM, Thompson BG, Taheri PA, Greenfield LJ: Antiplatelet therapy: an alternative to heparin for blunt carotid injury. J Trauma 52:896-901, 2002

\section{Response}

\section{William W. Scott, MD, and Kim L. Rickert, MD}

\section{Department of Neurosurgery, University of Texas Southwestern Medical} Center, Dallas, Texas

We would like to thank Drs. Heros and Peterson for their thoughtful comments on our paper. We agree that the increase in the number of recent papers devoted to this topic is indicative of the lack of knowledge surrounding the natural history and treatment of these injuries. The main risk of these injuries is thromboembolism, which Drs. Heros and Peterson point out. Our series demonstrated a much lower rate of stroke than most other series published, and although we cannot fully explain why this might be, this is one of the aspects that prompted us to evaluate the blunt traumatic carotid and vertebral artery injuries seen at our institution. The majority of the cerebral infarctions we identified were in patients presenting with stroke either on or very soon after admission, and we did not often see delayed strokes. Granted, although a significant proportion of the patients we studied who harbored these arterial injuries received a delayed head CT scan for various reasons, we did not routinely look for stroke with MRI 
in these patients, and therefore the editorialists' criticism about most patients not getting MRI studies is well taken. If we had obtained routine MRI studies in those patients, it is certainly possible that the number of identified strokes could have been higher than our reported incidence, but these infarctions would probably have been clinically silent and their significance unclear.

We typically treat all of our blunt vascular injuries with aspirin when it can be started. As expected within the trauma patient population, we did encounter individuals in whom aspirin could not be started right away. That said, it did not appear that these patients carried a higher risk of stroke; however, we have not adequately studied that question. Another question that needs to be answered is this: if most of the strokes occur prior to the vascular injury being discovered, how important is screening for these injuries? Are we just spending health care dollars that make no difference in patient outcome? In this day and age of attempting to cut the cost of medical treatment while balancing the risk of litigation, this surfaces as an important question. Hopefully, we can answer some of these questions in the future. 